\title{
Page-reRank: using trusted links to re-rank authority
}

\author{
Paolo Massa \\ ITC/iRst \\ Via Sommarive 14 - I-38050 Povo (TN) - Italy \\ massa@itc.it
}

\begin{abstract}
The basis of much of the intelligence on the Web is the hyperlink structure which represents an organising principle based on the human facility to be able to discriminate between relevant and irrelevant material. Second generation search engines like Google make use of this structure to infer the authority of particular web pages. However, the linking mechanism provided by HTML does not allow the author to express different types of links such as positive or negative endorsements of page content. Consequently, algorithms like PageRank produce rankings that do not capture the different intentions of web authors. In this paper, we review some of the initiatives for adding simple semantic extensions to the link mechanism. Using a large real world dataset, we demonstrate the different page rankings produced by considering extra semantic information in page links. We conclude that Web intelligence would benefit in adoption of languages that allow authors to easily encode simple semantic extensions to their hyperlinks.
\end{abstract}

\section{Introduction}

The idea of Web intelligence has been inextricably linked with the often described problem of information overload [17]. Early research on Web intelligence viewed the web as a large, unstructured, distributed data base where the goal was to index resources so that users could easily find them. As such, early search engines considered the topology of the web to be flat, where the goal was to crawl as much content as possible. However, the flat approach wouldn't allow search engines to discriminate between documents of varying quality, authority or relevance. An answer to this problem emerged when researchers began to consider the hyperlink structure of the web as a means of inferring page relevance. Whereas early search engines used links simply as means to find additional content to index, more recent algorithms, for example PageRank [14], weighted pages according to how many other pages linked to them. The key observation is that the hyperlink structure of the web represents an organising principle based on the human facility to be able to discriminate between relevant and irrelevant material. People who create web pages hand code links to other pages after judging the content on those pages. Thus, links explicitly characterize a distributed filtering of content by human intelligence. By tapping this intelligence, modern search engines such as Google (http://google.com) are able to return pages that not only match the submitted query but have been linked by many other pages in the web. As many of the current research initiatives on Web intelligence are based on mining the link structure of the web, in this paper we consider the semantics of using hyperlinks in web documents. We point out that a link may have several meanings: referential, endorsing or criticising. The unary nature of the link mechanism in the Hypertext Markup Language (HTML) means that these meanings cannot be communicated. For example, the PageRank algorithm considers all links into a page to be a vote for that page. However, this is not always the case and Google bombers and blog spammers have exploited this weakness in order to boost the PageRank score of their sites [5].

Consequently, we consider the semantics required for a new linking structure and examine several existing proposals for a richer linking structure for the Web. We argue that any change to the hyperlink facility must be comprehensible to the ordinary users of the Web. In order to test the effect of a more expressive linking structure, we examine the different rankings produced by PageRank for a large real world dataset where links are encoded using positive or negative trust statements. We see that the current unary link structure only allows PageRank to indicate how much attention is devoted to a particular page rather than how positively endorsed it is. We then consider what it means to have a graph based on negative links.

The paper is structured as follows. In Section 2 we give an overview of the hyperlink facility and describe the problems associated with it. In Section 3 we introduce and discuss various proposals intended to give web authors a more expressive linking language. In Section 4 we introduce the Epinions dataset and we describe our experiments on ranking using the PageRank algorithm. We discuss our results in Section 5. 


\section{Linking on the Web}

The term "hyperlink" was defined in 1965 by Ted Nelson during the Project Xanadu. In several books and articles published between 1964 and 1980, Nelson described his conception of a network of documents through which the reader makes her own trail by linking documents together. However, a research group led by Douglas Engelbart developed the oNLine System (NLS), the fist hyperlink system for connecting separate documents ${ }^{1}$. The precursor of the World Wide Web (WWW) was the Enquire programme developed by Tim Berners-Lee at CERN in 1980 in order to allow him to store and retrieve information about the structure of a system, such as the relationships and dependencies between people, programs, machines and ideas. The linking structure of Enquire allowed the author to define the type of relationship between two documents: such as part-of, made-by, similar-to. In order to extend the Enquire initiative to work in a shared multi-user information space, Berners-Lee developed the HTTP protocol and the HTML language. A crucial factor of the HTML language was its simplicity - a factor which allowed its rapid uptake. HTML contains only structural markup. The difficult task of agreeing a semantic mark-up language for the web was deferred. Likewise, the standard link semantics of the Enquire programme were discarded in favour of unary links that we have today on the Web.

In HTML, the anchor element $\langle a\rangle$ creates a link. The href attribute specifies the URL of the page resource being linked to. The description of the link known as anchor text is given between the opening and closing anchor elements.

\section{$\left\langle\right.$ a href $={ }^{\prime \prime} U R L "$ title $=$ "a hover box text" ${ }^{\prime \prime}$ link description $\langle/ a\rangle$}

Although, there are no predefined semantics for links in HTML, web authors generally use the anchor text to briefly describe the resource being linked to. We should note that some researchers have previously attempted to use the text that appears close to the link (anchor text) to predict some information about the link itself, for example what is the topic of the linked page in the opinion of the author of the link [6]. Google uses link description to enrich the description of the linked page for indexing [3]. In general, modern search engines attach a lot of weight to terms used in anchor text because they can represent a summary of the web author's impression or view of the web site. For example, they rank a page higher if all the sites that link to it use the same terms in their anchor text. This has led to a phenomenon known as Google Bombing [5] whereby specific anchor text is agreed in advance by a group of web authors linking to a site of a person or organisation. A query to Google using the anchor text will return the web page of the victim even

\footnotetext{
${ }^{1}$ http://sloan.stanford.edu/mousesite/1968Demo.html
}

though the page itself does not contain the query terms ${ }^{2}$.

So although the author can provide a free text description of the purpose of the link, machines are not sophisticated enough to detect when the author is being truthful, playful or malicious. This is problematic because the second generation of search engines such as Google, rely upon the link structure of the web to infer quality and relevance of search results. In the next section we look at some proposals for providing machine readable semantics to hyperlinks and we discuss the requirements and implications of such proposals.

\section{Current Proposals}

The second generation search engines have been able to improve search performance by considering and exploiting the hyperlink structure of the Web. PageRank [14], one of the algorithms powering Google, was the first with notable success in doing this. The basic idea of PageRank is that if page $A$ links to page $B$, then the creator of page $A$ is implicitly assigning some importance to page $B$. The PageRank or importance of page $A$ depends on the number of pages linking to it and on their importance. Intuitively, if many important pages point to a certain page, that page should be important as well. The notion of importance in PageRank is based simply on a measure of its 'connectedness' within the graph of the World Wide Web. Any semantics attached to the links by the author are not considered. An intuitive justification of PageRank is that it is the probability that a surfer, starting at a random page and choosing a links at random, will visit that page [3]. The randomness of the surfer's behaviour illustrates the insensitivity of the algorithm to the intentions of web authors. Thus a high PageRank score does not necessarily always correspond to positive endorsement. Authors can link to a page because they disapprove of the content expressed there and they may want to comment upon it: for example, linking to the web site of a disliked political candidate or a site with offensive or controversial content. In this cases, a link may simply be referential or constitute a negative endorsement.

However, it seems that humans tend to speak more about what they like and less about what they don't like (we provide some empirical evidence of it in Section 4). For this reason, the adopted heuristics works most of the time. Nevertheless, as web authors are often aware of increasing the PageRank of a page they link to, there is an incentive not to link to pages they disagree with. In this sense, the limited expressiveness of the language may be affecting the way authors encode their beliefs and opinions. We would argue that, in order to foster discussion and creativity, it is necessary to express both positive and negative opinions as well as the reasons for such opinions.However at the moment it

\footnotetext{
${ }^{2} \mathrm{~A}$ list of successful Google Bombs is given here: http://en.wikipedia.org/wiki/Google_Bomb
} 
is not possible for an author to express a machine readable "semantics" for the link. In the following we will review some current initiatives to express "semantics" with links.

\subsection{Introducing Link Semantics}

VoteLinks [16] is a microformat created by Technorati (http://technorati.com) in which they propose a set of three new values for the rev attribute of the a tag in HTML. The values are vote-for, vote-abstain and vote-against and represent agreement, abstention or indifference, and disagreement respectively. For example, with the following HTML code the author of a page can link to a site in order to criticise it and, in doing so, communicate (to search engines but also to human readers) that she disagrees with it.

\section{$\left\langle\right.$ a rev $=^{\prime \prime}$ vote-against" href $={ }^{\prime \prime}$ http://example.com" $\rangle$ bad site $\langle/ a\rangle$}

Although, the proposal was made in early 2004, it seems the adoption of this microformat is minimal. By Nov 26, 2004, Technorati had aggregated only 299 VoteLinks from 157 pages $^{3}$. At this time, the sites of the two US presidential candidates received the greatest number of VoteLinks. An important point of VoteLinks proposal is that the specification is very simple so that humans can understand, remember and use it easily.

XFN (Xhtml Friends Network) [9] is a similar initiative which proposes a set of values for the rel attribute of the a HTML tag. XFN is a microformat as well and was developed by the Global Multimedia Protocol Group. Using the proposed values, web authors can represent their relationships to other people on the Web such as, for example, friend, colleague, neighbor, sibling, spouse, date. This microformat is supported and adopted by the users of the popular blogging tool Wordpress (released as Free Software and downloadable at wordpress.org).

Another initiative has recently been proposed by Google and quickly embraced by many other search engines and blogging tool creators. Google suggested to add rel="nofollow" [8] to a hyperlink in order to indicate that the destination of that hyperlink should not be afforded any additional weight or ranking by user agents which perform link analysis upon web pages (for example, search engines). The motivation for this proposal is the problem of blog and wiki spammers: automatic programs that post on blogs comments containing links or edit wiki pages adding links in order to manipulate PageRank into assigning a high rank to certain linked web site. Google proposes that blogging and wiki tools convert the links found in comments posted by all users by adding the rel="nofollow" attribute to the corresponding $\langle a\rangle$ tag. The rationale is that links appearing in comments to blog posts are not created by the owner of the blog and therefore should not improve the rank of the linked page by drawing upon the rank or importance of the blogger. A similar argument applies for links inserted into wikis. The rel="nofollow" attribute instructs search engines not to consider the link as an expression of the opinion of the author of linking page. Thus, it is similar to rel="vote-abstain" in VoteLinks but has received greater recognition because of Google's position as the most popular search engine on the Web. A drawback is that it does not allow the user to express a negative preference for a link.

\subsection{Adoption Issues}

All these proposals consider adding machine readable semantics to the existing linking structure of the web. Likewise, Semantic Web researchers are involved in defining precise and complex protocols and languages to allow web authors to unambiguously express the content and the relations and semantics attached to the content. [2].

It remains to be seen how many of the standards developed as part of this project will be adopted or whether web authors will continue to adopt and embrace simple incremental improvements. For example, the World Wide Web Consortium (W3C) recommended, on 27 June 2001, the XML Linking Language (XLink) ${ }^{4}$. The goal is to allow authors to describe both basic unidirectional links and more complex linking structures. Its reduced adoption seems to suggest that the specification is too complicated to be understood and used by web authors. Instead, we suggest that initiatives, such as the two microformats mentioned earlier, that rely on simple incremental changes on top of currently widely used protocols are more likely to meet with success.

However, while the $\mathrm{W} 3 \mathrm{C}$ is in charge of defining de jure standards for the Web, it is clear that certain companies (or even single individuals) have the clout to propose and consolidate new de facto standards. For example, Google's rel=" nofollow" attribute was widely adopted in few weeks. On the other hand, the microformats we reviewed previously have had less success in getting a wide adoption, despite their usefulness and intrisic quality.

A key factor, of course, is the ease with which the new improvements can be understood by user and the support offered by related software devices. Since most authors nowadays do not directly write HTML code, WYSIWYG HTML editing software would need to enable the author to express semantic values for the created link. For example they might give the user the possibility to "vote-for", "voteagainst", "vote-abstain" with a simple click and possibly leave as default the "vote-abstain" or "vote-for" depending on the application. Also Web browsers would need to display the semantics of the link. There are many ways of doing this, such as with an extension or plugin for the browser or styling the site using Cascading Style Sheets (CSS) ${ }^{5}$

\footnotetext{
${ }^{4}$ http://www.w3.org/TR/xlink/
}

${ }^{3} \mathrm{~A}$ version of the page archived on Nov 26, 2004 is at http://web.archive.org/web/20041126091750/http://technorati.com/live/votes.html ${ }^{5}$ To illustrate, we have modified the TargetAlert exten- 
Of course having the "semantics" of the link unambiguously expressed by the author would allow more reliable predictions, results and new algorithms.

\section{Experiments}

Although ranking algorithms may be able to find important pages (pages linked by many other pages and hence receiving a lot of attention), they cannot distinguish between positively and negatively endorsed pages. In this section, we present some experiments on real world data that demonstrate this intuition.

We describe firstly Epinions (http://epinions.com), the provider of the data, and then the experiments we have run on this data.

\subsection{Epinions Dataset}

Epinions is a web site where registered users can write reviews about products (such as books, movies, electronic appliances, restaurants, software, ...) and also assign them numeric ratings. Epinions also allows the user to explicitly designate the users she trusts (i.e. reviewers whose reviews and ratings they have consistently found to be valuable) and the users she distrusts (authors whose reviews she finds consistently offensive, inaccurate, or in general not valuable) [7]. Based on this information, the system can provide personalized views to every user by boosting reviews of trusted users and suppressing reviews of distrusted users. For our experiment, we make a straightforward analogy with the web: web pages are Epinions users and links are trust and distrust statements (see Figure 1). The relevant difference with the Web is that Epinions allows the expression of positive (trust) and negative (distrust) links. Again, the parallel with the VoteLinks proposal is straightforward: trust statements are vote-for links and distrust statements are vote-against links.

Let us now analyze the structure of the directed graph representing Epinions community. The data consists of 131,828 nodes and 841,372 edges. All the information is anonymized so that nodes are represented by obscure numeric identifiers. The edges are labeled either as 'trust' or 'distrust'. In Figure 1, trust and distrust statements are represented respectively by $\mathrm{a}+$ and $\mathrm{a}-$ near the edge. Precisely, $85.29 \%$ of the edges are labeled trust and only $14.71 \%$ are distrust edges. Some statistics about this graph can be found in [10]. In particular, the in-degree and outdegree distributions of this directed graph, treating both the trust and distrust edges, suggest a power law typical of the structure of the Web. However, the exponent of Epinions graph is -1.7 while, in general, the various power laws that

sion for Mozilla Firefox in order to show a thumb-up/thumbdown/thumb-null for rel="vote-for/vote-against/vote-abstain" The new extension, called SemanticLink, can be found at http://moloko.itc.it/paoloblog/semantic_link.html.

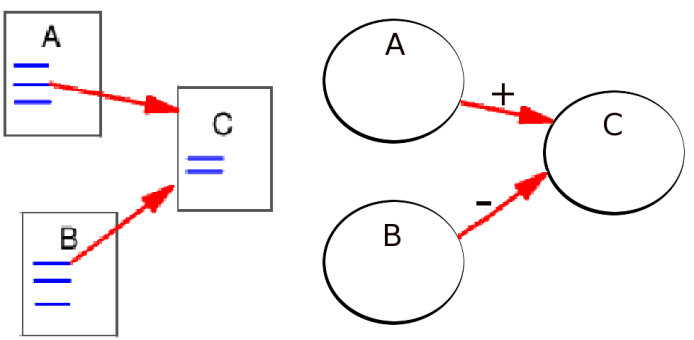

Figure 1. On the left, Web page $A$ and Web page $B$ link to Web page $C$. On the right, Epinions user $A$ and Epinions user $B$ judge Epinions user $C$. The graph structure is the same. Note that A trusts $\mathrm{C}$ while B distrusts $\mathrm{C}$.

have been observed on the Web present exponents lower than -2.0 . For an analysis of the exponents of different networks see Table 2 of [13]. This graph presents other characteristics similar to the ones observed for the Web, for example the existence of a single large strongly connected component and the bow tie structure [4], as reported in [10].

Moreover, the fraction of trust and distrust links confirm our intuition: humans tend to refer more to what they appreciate and less to what they don't appreciate. Of course, the user interface of Epinions has an heavy impact on this fraction as well.

\subsection{Experimental Setup}

In this paper we claim that search engines could produce different rankings if they were able to consider the expressed intentions of web authors. At present, the link structure loses this information. In order to demonstrate our claim, we have run the PageRank algorithm against two versions of the Epinions graph. In the first setting, we have considered all the statements (both trust and distrust) as links between users. This is the information that can be expressed on the Web at the moment as there is no way in HTML to attach semantics to the link. In the second setting, we build a graph by keeping only the trust edges. In this way, we consider only the expressions of positive endorsement between users. In the following, the first setting is called $p r+/-$ and the second is called $p r+$. In terms of the VoteLinks proposal, this would be the same as propagating reputation along both "vote-for" and "vote-against" $(p r+/-)$ or only along "vote-for" links $(p r+)$. We have also run PageRank on the graph composed only of distrust edges $(p r-)$. We will comment on this later in the section.

\subsection{Results}

The returned ordered lists are shown in Figure 2. The first two represent, respectively, attention ( $p r+/-)$ and trust 


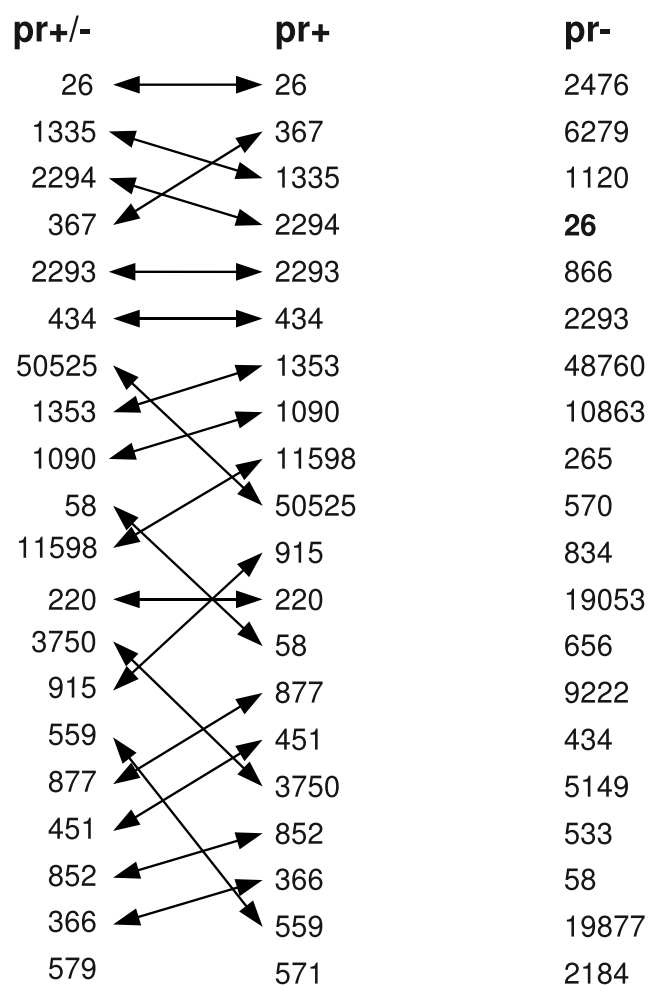

Figure 2. Top20 ranked lists of Epinions users representing (left) "attention" and (center) "trust". The attention list is obtained running PageRank considering as links both trust and distrust edges, while the trust list is obtained running PageRank considering only trust edges. The rightmost list is obtained running PageRank considering as links only distrust edges.

$(p r+)$ and we will refer to them as "attention list" and "trust list". Note that, while it is possible to have a highly disliked page on top of the first list (as long as it received many links), this cannot happen on the second list that propagates only along the "vote-for" (positive) links.

User 367 is $4 t h$ in the attention list and $2 n d$ in the trust list; this means that users 1335 and 2294 are more "spoken about" (considering both trust and distrust) while, if we consider only trust statements, user 367 is better placed. A search engine that wants to return the most appreciated users (or pages in the analogy) should probably return 367 before 1335 and 2294. An opposite argument can be given for user 50525: she is $7 t h$ in the attention list but only $10 t h$ in the trust list. This means that, while she is known and judged (linked to) by many users, there is a non negligible portion of them that distrust her. Again, in this case, a search engine that wants to return mainly trusted pages should give preferences for example to 1353, 1090 and

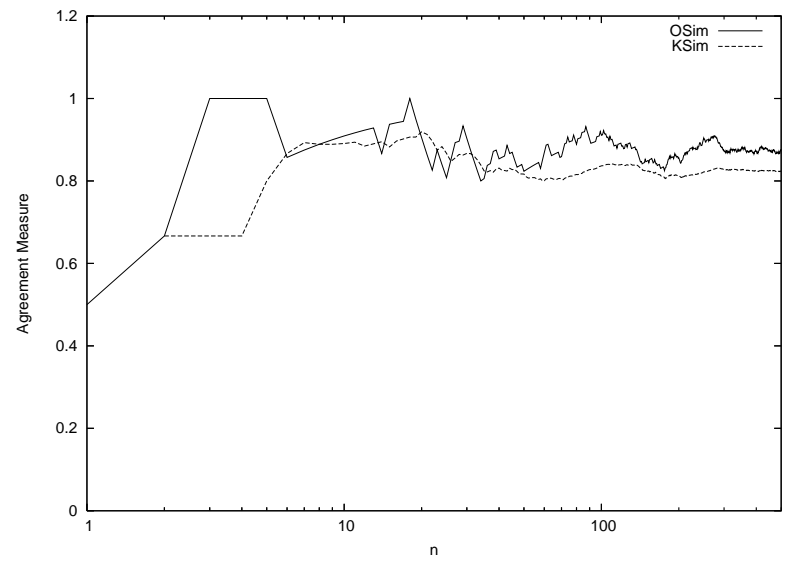

Figure 3. OSim and KSim of the two Top $n$ lists generated by $p r+/-$ and $p r+$. The $\mathbf{x}$-axis is in logarithmic scale to give more visibility to small values of $n$.

11598.

In order to better appreciate the differences produced by the two languages we have also computed two measures of misalignment of the two lists as defined in [12]. The first measure, denoted $\operatorname{OSim}_{n}\left(\tau_{1}, \tau_{2}\right)$, represents the degree of overlapping between the top $n$ elements of two rankings, $\tau_{1}$ and $\tau_{2}$. The overlap of the two sets $A$ and $B$ (each of size $n$ ) is defined to be $\frac{|A \cap B|}{n}$ so that OSim is in the interval $[0,1]$ where 1 means total overlapping. The overlap measure OSim does not give a complete picture of the similarity of two rankings, as it does not indicate the degree of agreement between the relative orderings of the top $n$ users produced by two different rankings.

Therefore, we also use a variant of the Kendall's $\tau$ distance measure. For consistency with OSim, it is defined as a similarity (rather than a distance) measure, so that values closer to 1 indicate closer agreement. In short, $\operatorname{KSim}\left(\tau_{1}, \tau_{2}\right)$ is the probability that $\tau_{1}^{\prime}$ and $\tau_{2}^{\prime}$ agree on the relative ordering of a randomly selected pair of distinct nodes $(u, v) \in U \times U$. The precise definition of OSim and $K S i m$ can be found in [12].

Figure 3 plots the two similarity measures when comparing the lists returned by $p r+/-$ and $p r+$, while Figure 4 considers $p r+/$ - and $p r$ -

\subsubsection{Analysis of results}

Figure 3 clearly shows the amount of disagreement between the attention list and the trust list. The information presented verbosely in Figure 2 is here presented in a compact form. The alignment tends to be a little bit greater than 0.8 (both when computed with OSim and KSim). We believe that this is an important fact. The difference between the attention list (what search engines can return at present) and 


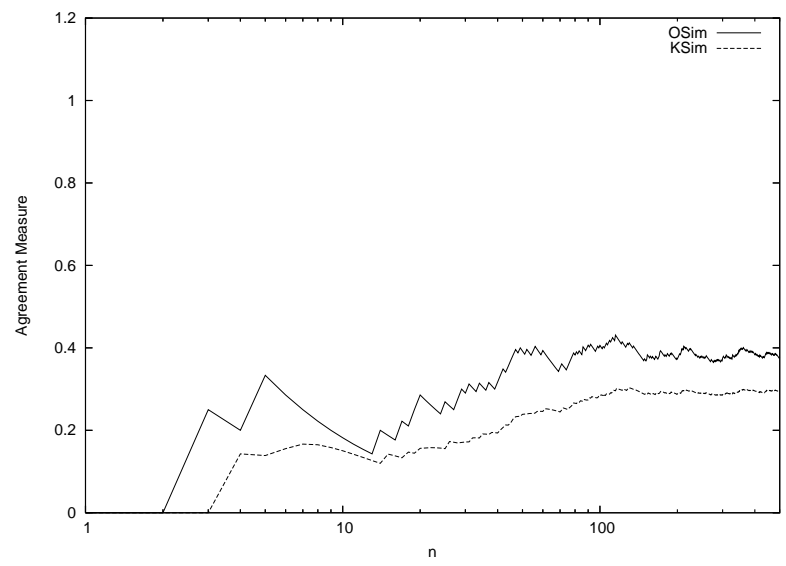

\section{Figure 4. OSim and KSim of the two Top $n$ lists generated by $p r+/-$ and $p r$-. The $\mathbf{x}$-axis is in logarithmic scale to give more visibility to small values of $n$.}

the trust list (a list that would correspond to most appreciated pages) is significant.

When we are to compare the attention list with the list returned by $p r$ - (Figure 4), we observe, as expected, that the alignment is much less. While comparing Top $n$ lists for small values of $n$, it is very small. However note that already for Top 50 lists, OSim is around 0.4, indicating that the overlapping between the attention list and $p r$ - list is not so small. This means that in the attention list there are many users that are also in the list obtained by propagating reputation along negative links. This fact is far from ideal if a search would like to return only or mainly highly appreciated pages.

\subsubsection{Distrust Links}

Let us now analyze $p r$ - that is PageRank algorithm run on the graph composed only of negative links. In this case PageRank would propagate "negative" authority along links. But PageRank intuition is not really meaningful in this context. If user $A$ distrusts user $B$ and user $B$ distrusts user $C$, this does not mean that user $A$ would necessarily distrust user $C$. In some situations, the opposite can even be true. In short, propagating distrust does not make sense and it is interesting to note how the intuition behind PageRank, propagation along links, totally loses its meaning in a context in which links represent negative statements of distrust.

For this reason, $p r$ - Top 20 list (rightmost column of Figure 2) does not represent a list of most distrusted users and actually it is not clear what this list represents. However, let us examine it. The users in the $p r$ - Top 20 list are very different from the users in the other two lists. The only user present in all of them is user 26 , that happens to be an user with a very large number of incoming edges (both trust and distrust). In the real world, she would have been what we call a controversial star, known by almost everyone, appreciated by many but also criticised by many. The other users in the $p r$ - list, instead, received mainly negative links. A short discussion about how the issues of utilizing "voteagainst" information to produce more meaningful rankings is presented in Section 5.

\section{Discussion}

The ranked lists returned by $p r+$ and $p r+/$ - (Figure 2) are different and represent different concepts: trust and attention, respectively.

However, at present, search engines can only return the attention list because trust and distrust statements cannot be unambiguously expressed in HTML. For example, during election time, search engines are able to return the Web sites of the candidates that are heavily discussed but cannot return the ones who are most trusted and appreciated.

We think that it is important to be able to have such a distinction and we support the VoteLinks microformat: users should be able to express their opinion when they link to a page. Of course, the adoption of such a microformat would be easier and wider if HTML editors (and weblogging tools) were to make this feature available to normal users with a single click. We believe this is a correct first step to enriching links with semantics.

Since humans tend to mention what they like more than what they don't like, the $p r+$ and $p r+/-$ are largely overlapping and aligned. However, there are some users who have different positions in the two lists and this information is lost with the current HTML language.

Another point worth discussing is about $p r-$, that is applying PageRank to the graph composed just of distrust statements. The intuition behind PageRank in the context of negative links ("A negative authority is a page linked by many negative authorities") does not make much sense. The user distrusted by the most distrusted user is not necessarily the most distrusted. While trust is in some sense transitive and can be propagated, distrust is certainly not transitive [10].

Social balance theory [15] states that "my enemy's enemy is my friend" but this is not really always the case. Our opinion is that if $A$ distrusts $B$ then $B$ should not at all influence the opinions of $A$ about other users or items, otherwise $B$ could express her opinions in an instrumental way in order to influence recommendations to $A$ : from the point of view of $A$, opinions of $B$ should simply be ignored.

Instead, in this context, a simple adaptation of PageRank intuition is that a negative authority is a page judged as negative by many positive authorities. Hence, it makes sense to propagate authority along vote-for links in order to discover positive authorities $(\mathrm{pr}+)$. Then using vote-against 
only in the last step, assuming that a page voted-against by many positive authorities is a negative authority.

However, this is still open to abuse. A site with a high positive PageRank is an authority, since many pages link to it. Such a site has a lot of PageRank weight when it comes to assigning negative links. If this site negatively links to a smaller organisation, the smaller organisation will suffer in terms of its ranking. In the worst case scenario, a dominant organisation or interest group could use its positive PageRank to suppress opposition or competition. This type of behaviour could lead to negative link 'flame' wars where rival interest groups try to damage each others' rank.

However the goal of this paper is not to discuss how the semantic information encoded by authors along their links could be used but just to suggest to the Web Intelligence research community that a preliminary requirement must first be accomplished: extending the Web language so that semantic links can be expressed. [10] provides an analysis of different ways to propagate trust and distrust. Moreover, several algorithms have been proposed that exploit weighted relationships between nodes in a graph in order to infer authority and reputation, for example Trust Metrics [18, 1]. A logical next step for search engines is to produce ranked lists that are personalized to the opinions of the specific active user $[11,12]$. In this context, links that express positive and negative preferences open new interesting possibilities.

Summarizing, attention is not always a synonym of trust. We have shown that in fact, on a real world dataset, the two ranked lists representing these concepts are different. This fact clearly show that enriching HTML so that it is possible to represent some simple semantics along with links would benefit the research community and the web users at large.

\section{Conclusions}

In this paper we consider the potential for an extended linking language on the Web. Although search engines like Google tap the intelligence hand coded into the link structure of the Web, they are constrained from extracting more information from links by the lack of semantics available in the current linking model. We review several current proposals for extending the link mechanism and observe that any extension must be easy to use and be serviced by an intuitive tool set. Using a real world dataset from Epinions as a a proxy for the Web, we demonstrate how additional link information would allow Google's PageRank algorithm identify highly trusted web sites. We conclude that simple semantic extensions to the link mechanism would provide a richer semantic network from which to mine more precise Web Intelligence.

\section{Acknowledgment}

The authors would like to thank Epinions for kindly making available the data for this study. We would in particular like to thank also R. Guha, Ravi Kumar, Prabhakar Raghavan and Andrew Tomkins. We would also like to thank Michelangelo Diligenti of Google for sharing his implementation of PageRank code.

\section{Licence}

This work is licensed under the Creative Commons Attribution-ShareAlike 2.5 License. To view a copy of this license, visit http://creativecommons.org/licenses/by$\mathrm{sa} / 2.5 /$ or send a letter to Creative Commons, 543 Howard Street, 5th Floor, San Francisco, California, 94105, USA.

\section{References}

[1] P. Avesani, P. Massa, and R. Tiella. Moleskiing: a Trustaware decentralized recommender system. In Proceedings of the 1st Workshop on Friend of a Friend, Social Networking and the (Semantic) Web. Galway, Ireland. 2004

[2] T. Berners-Lee, J. Hendler, and O. Lassila. The semantic web. Scientific American, 2001.

[3] S. Brin and L. Page. The anatomy of a large-scale hypertextual web search engine. In WWW7: Proceedings of the seventh international conference on World Wide Web 7. Elsevier Science Publishers B. V., 1998.

[4] A. Broder, R. Kumar, F. Maghoul, P. Raghavan, S. Rajagopalan, R. Stata, A. Tomkins, and J. Wiener. Graph structure in the Web. Computer Networks, June 2000.

[5] S. Byrne. Stop worrying and learn to love the google-bomb. Fibreculture Journal, issue 3.

[6] S. Chakrabarti, B. Dom, P. Raghavan, S. Rajagopalan, D. Gibson, and J. Kleinberg. Automatic resource compilation by analyzing hyperlink structure and associated text. In WWW7: Proceedings of the seventh international conference on World Wide Web 7. Elsevier Science Publishers B. V., 1998.

[7] Epinions.com web of trust faq. Retrieved December 28, 2005, from http://www.epinions.com/help/faq/?show=faq_wot.

[8] Google. Preventing comment spam, 2005. http://googleblog.blogspot.com/2005/01/preventingcomment-spam.html.

[9] G. M. P. Group. XFN (Xhtml Friends Network). http://gmpg.org/xfn/.

[10] R. Guha, R. Kumar, P. Raghavan, and A. Tomkins. Propagation of trust and distrust. In WWW'04: Proc. of the 13th int. conf. on World Wide Web, pages 403-412. ACM Press, 2004.

[11] T. Haveliwala, S. Kamvar, and G. Jeh. An analytical comparison of approaches to personalizing pagerank, 2003.

[12] T. H. Haveliwala. Topic-sensitive pagerank. In $W W W$ '02: Proc. of the 11th int. conference on World Wide Web, pages 517-526, New York, NY, USA, 2002. ACM Press. 
[13] M. E. J. Newman. The structure and function of complex networks. SIAM Review, 45:167-256, 2003.

[14] L. Page, S. Brin, R. Motwani, and T. Winograd. The pagerank citation ranking: Bringing order to the web. Technical report, Stanford, USA, 1998.

[15] H. Situngkir and D. Khanafiah. Social balance theory: Revisiting Heider's Balance Theory for many agents. Technical Report 0405004, Bandung Fe Institute, Indonesia, May 2004. available at http://ideas.repec.org/p/wpa/wuwpio/0405004.html.

[16] Technorati.com.

VoteLinks. http://microformats.org/wiki/vote-links.

[17] A. Toffler. Future shock, 1970. Random House, New York.

[18] C. Ziegler and G. Lausen. Spreading activation models for trust propagation. In IEEE International Conference on eTechnology, e-Commerce, and e-Service (EEE'04), 2004. 